\title{
Automated brain tumor classification using various deep learning models: a comparative study
}

\author{
Alaa Ahmed Abbood ${ }^{1}$, Qahtan Makki Shallal ${ }^{2}$, Mohammed A. Fadhel ${ }^{3}$ \\ ${ }^{1}$ Faculty of Business Informatics, University of Information Technology and Communications (UOITC), Baghdad, Iraq \\ ${ }^{2}$ Management Technical College of Basra, Southern Technical University, Basra, Iraq \\ ${ }^{3}$ College of Computer Science and Information Technology, University of Sumer, Thi Qar, Iraq
}

\begin{tabular}{l} 
Article Info \\
\hline Article history: \\
Received Oct 11, 2020 \\
Revised Jan 27, 2021 \\
Accepted Mar 2, 2021 \\
\hline
\end{tabular}

\section{Keywords:}

Accuracy

Brain tumor

Deep learning

GPU

Processing time

\begin{abstract}
The brain tumor, the most common and aggressive disease, leads to a very shorter lifespan. Thus, planning treatments is a crucial step in improving a patient's quality of life. In general, several image techniques such as CT, MRI, and ultrasound have been used for assessing tumors in the prostate, breast, lung and brain. Primarily, MRI images are applied to detect tumors in the brain during this work. The enormous amount of data produced by the MRI scan thwarts tumor vs. non-tumor manual classification at a particular time. Unfortunately, with a small number of images, it has certain limitations (i.e., precise quantitative measurements). Therefore, an automated classification system is necessary to avoid human mortality. The automatic categorization of brain tumors in the surrounding tumor region is a challenging task concerning space and structural variability. Four deep learning models: AlexNet, VGG16, GoogleNet, and RestNet50, are used in this comparative study to classify brain tumors. Based on accuracy, the results showed that RestNet50 is the best model with an accuracy of $95.8 \%$, while AlexNet has the fast performance with a processing time of 1.2 seconds. In addition, a hardware parallel processing unit (GPU) is employed for real-time purposes, where AlexNet (the fastest model) has a processing time of only $8.3 \mathrm{msec}$.
\end{abstract}

This is an open access article under the $\underline{C C B Y-S A}$ license.

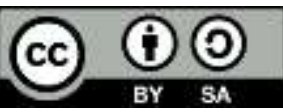

\section{Corresponding Author:}

Mohammed A. Fadhel

College of Computer Science and Information Technology

University of Sumer, Thi Qar, Iraq

Email: mohammed.a.fadhel@uoitc.edu.iq

\section{INTRODUCTION}

The human brain is one of the most complicated and functional units that control the entire human body. Unfortunately, the brain cells under certain conditions got uncontrolled division due to unknown reason creating an abnormal group of cells surrounding or inside human brain named as "tumor" [1]. Therefore, these abnormal cells categorized depending on their initial origin into metastatic brain tumors and primary brain tumors. The primary brain tumors originated from the brain tissue, while the metastatic caused by cells cancerous growth in different places of the human body cells and then expand to the brain. Consequently, both tumor types affect the main brain functionality through damaging the healthy cells [1-2]. Medically, they recognized the brain tumor as a low-grade called "benign" and high-grade named "malignant" [1]. The Benign tumors also termed as (non-cancerous) which is originated from the brain cells with gradual growth and considered to be less aggressive with limit spread to anywhere else in the body. While, the malignant tumors which is considered as cancerous with rapidly growth forming unknown boundaries with high prevalence over all of the human body [3-4]. 
The patient with a symptom of the brain tumor goes through several biological and radiological tests to diagnose the tumor position and their type. The most common radiology tests used to inspect brain tumors are PET (positron emission tomography), SPECT (single photon emission computed tomography), MRI (magnetic resonance imaging), and CT (computed tomography). Medically proven, the MRI considers as one of the best imaging techniques used for demonstrating the tumor in the identification and treatment stages [34]. MR imaging has an advantage over other imaging systems is that it doesn't emanate any unsafe radiation to the human body [3,5]. Furthermore, the MRI technique provides rich information on the brain tissues structure with high-quality images to visualizing the abnormalities in the brain tissues [5]. The accurate diagnosis with the MRI technique shows all the anatomical information of the human brain that guides to achieve an accurate diagnosis and treatment of the brain tumor.

Currently, many classification approaches are presented to categorize MRI brain images such as KNN (k-nearest neighbor) neural networks, fuzzy logic, rule-based techniques and SVM (support vector machine) [6-9]. In addition, the deep learning approach (DL), which is a machine learning technique, is used as an efficient technique to classify the brain tumor $[4,2,10]$. Therefore, our contributions are summarized as follows:

a) This paper takes advantage of a deep learning algorithm to perform automatic classification of MRI brain images.

b) The current methodology intends to measure the performance of various deep learning models with different platforms to classify brain tumors.

c) The outline of this paper includes compared by four popular deep learning models, AlexNet, VGG16, GoogleNet, and RestNet50 in term of accuracy and time of processing in different platforms.

\section{RELATED WORK}

Radiologists investigate the MR slices through visual inspection to distinguish and recognize the available tumor or irregular growth tissues. The huge number of the MRI slices makes the traditional processes with visual inspection interspersed with labor-intensive, costly, and often erroneous. Moreover, in some cases especially when less available information on MRI slices for the influenced region lead to reduce the sensitivity of the human eye during inspections. Therefore, an automated analysis and classification system with use of computer algorithm is essential to diagnose the tumor in MRI brain slices. Automatic MR brain image classification techniques have been widely investigated during the last decade. Over the years, a number of different classification techniques have been developed using multiple classification approaches and datasets. Each classification method has its own specific characteristics that researchers capitalize on to advance classification research using a particular dataset $[3,11]$. The most common datasets, widely used by the researchers, are summarized as follow: (I) IBSR (internet brain segmentation repository) (10Normals_T1) devoid of brain tumor generated in USA by the massachusetts general hospital, center for morphometric analysis, (II) IBSR (536_T1) contains brain tumor (III) multimodal brain tumor segmentation (BRATS) challenge dataset [11].

In 2010 the study of [12] applied the SVM classifications technique and created a system that able to categorize the MRI brain tumor into either benign or malignant. They used FPGA (field programmable gate array) device for managing the data. On the other hand, multiple phases are proposed for diagnosing the MRI brain tumor, starting with the texture feature extraction that uses for the classifications, followed by the ensemble base classifier and finally the segmentation phase. The implemented methodology was able to detect normal and abnormal MRI brain tumor with an accuracy reached up to 99\% [13]. Kharrat, with his colleagues in 2010, proposed automatic MRI brain tumor classifications by implementing the WT (wavelets transform) as input to genetic algorithm (GA) and SVM. Their experimental result achieved a significant rate [14]. One year later, N. Hema Rajini and R. Bhavani in 2011 proposed hybrid classification scheme by merging k-NN and ANN (artificial neural network) algorithms. The proposed technique goes through two stages: the features extracted by applying the DWT (discrete wavelet transform) technique in the first stage. The second stage represents the classification technique. Their classification approach consists of forwarding ANN and k-NN classifiers. The proposed classifiers approach come out with an accuracy of 90\%, using FP-ANN classifier, and reaches up to $99 \%$ with $\mathrm{k}-\mathrm{NN}$ classifier.

Later in 2015, improved particle swarm optimization (IPSO) classification approach was introduced by [15]. The study involved a pre-processing technique, which includes image segmentation, feature extraction, and feature selection. Some emendations to the dynamic classifier selection and the dynamic local training were made to form combinations of multi-classifiers for the final decision [16]. Their technique was examined on 20 MRI, from the IBSR dataset, and they showed a sufficient classification result. Chandan Saha and Md. Faisal Hossain, in 2017 used K-Means Clustering, NSCT, and SVM as classifiers to design fully automatic MRI brain tumor classification scheme. The suggested system enhanced the MRI brain images by applying a 
median filter. Then, image segmentation was performed with K-means clustering. Subsequently, the classification features were extracted by using NSCT coefficients. Finally, the extracted features are fed to the SVM classifier of MRI brain images as abnormal, if the MRI slice contains tumor otherwise, benign [17]. Basically, most of the previous methodologies shares similar process pipeline through implementing same process phases like pre-processing, followed by feature extraction, and finally applying classification techniques. Moreover, different algorithms were applied to improve the MR brain image quality. Later, the feature's phase that differentiates all the tissues in the MRI brain were extracted. Some of the most common MRI brain features that were used in previous studies include: edge-based, intensity gradients, local image textures, asymmetry-related features, textons, first-order statistical features, multifractal Brownian motion feature, variance intensity, and discrete wavelet transforms DWT features [1-4, 6, 18, 19]. Finally, different classification approach proposed based on the extracted features included neural networks (NN) classifier, SVM classifier, k-NN classifier, self-organizing maps (SOM), machine learning ML and deep learning DL classifiers $[1,2,4,6,9,15,17,18]$. Despite several notable contributions, there remain potentially new findings in this area. Many issues related to MRI brain tumor classification, as well as image pre-processing, are being resolved. However, the following key area is worth looking at.

\section{OVERVIEW ON DEEP LEARNING}

Deep learning technique is an advanced machine-learning algorithm, designed by hierarchically representing the system's features. The structure of the DL system works as that the top-level features formed by the bottom level features, and the bottom level features able to generate many other top-level features and so on [4]. Practically proven that the obtained results from the DL technique are higher performance than that of the other machine learning algorithms, especially when applied for large dataset [20]. The DL technique considers closest to mimics the human brain functionalities [21]. In the recent studies, the invention of deep learning techniques rapidly expands the use of artificial intelligence in pattern recognition, image segmentation, and classifications [22-24]. On the other hand, Gatys and his colleagues implemented the deep learning algorithm in Artistic Style to generate images with different styles [25].

The convolutional neural networks (CNNs), which considered as a well-known architecture of DL, were able to perform complex processes with the help of the convolution filter. The data in CNN's were processed as multiple arrays; for example, the array of $3 \times 2 \mathrm{D}$ represents the values of different pixel intensities in a grey-scale image. The CNNs are able to use the properties of the natural signals through four ways, which include pooling, local connections, shared weights, and utilization of different layers [4]. Graphically, Figure 1 describes the CNNs architecture, where at the beginning, only two layers are created termed as convolutional and pooling layers. The elements within the first layer organized as feature maps, each element linked to the nearby fixes of the feature maps from the previous layers through weights. All the components in the feature map share a comparative channel bank, while different feature maps inside the layer utilize distinctive channel banks. From Figure 1, clearly seen there are two objectives of using this architecture. First, investigating the local area through their values and easily detecting the related units. Second, invariability of the local statistical analysis values. From the mentioned objectives, if the detected motifs from a part of an image, then it may present elsewhere on the same image and sharing the same weights. The convolution layer identifies the local pattern of the feature based on the previous layer while the pooling layer merges of similar features into a single feature.

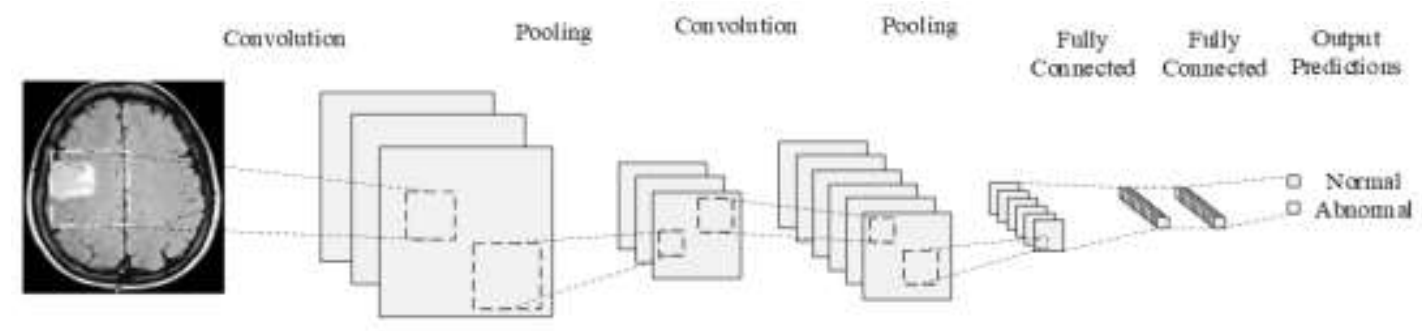

Figure 1. Image classification based on the CNN

In the study of Chen et al. [26], CNN's method was applied for facial classification based on edge detection. In the beginning, the raw pixels are presented in the layer 1 of CNNs to identify the edges. The simple shape detection and the high-level feature recognition were performed on layer two based on the information 
obtained from layer1. Lastly, these high-level features are used in the final layer to classify the facial feature. Generally, the CNNs consist of many layers named as hidden, output, and input layers. The hidden layers might conventional layer, pooling layer, or completely connected. The procedures and functionalities of these layers will describe in the next section.

\subsection{Convolutional layer}

The convolutional layer is an essential part of $\mathrm{CNN}$, which is operated by using the convolution operation as input and then pass the output to the consequent layer. The convolutional layer consists of many filters that take the same size and dimension of the input image. In addition, local connectivity and parameter sharing are two concepts used to reduce the number of model parameters. In the local connectivity concept, the whole neurons in the feature map are associated with the local neuronal patch of the earlier layer. While, in the parameter sharing concept, the neurons in the feature map were having similar parameters, that made all the neurons in the feature map scan for the same features of the earlier layers at different areas of the image. Hence, the variety of the feature map enables it to identify the edges at many locations within the image. The neuronal activity of the convolutional layer is calculated using a discrete convolution filter [27].

The size of the output is measured using the 3D array termed as zero padding, stride, and depth. The depth shows the quantity of filters that were applied, such as filters used for detecting blobs, corners, and edges. The stride indicates, the quantity of pixels went through the filter during sliding over an image. Lastly, zero padding denotes the filling of 0 s around an input image's boundary for keeping its size.

\subsection{Rectified linear units (ReLU) layer}

The convolutional layer directly followed by a nonlinear layer or an activation layer [28]. This layer presents nonlinearity to a system that computes the linear processes in the convolutional layers. The ReLU layers proven an efficient performance due to the network could be trained quickly, without influence its accuracy. The ReLU layer used $\mathrm{f}(\mathrm{x})=\max (0, \mathrm{x})$ function to input values. Thus, this layer-able to increase the negative values to 0 [29]. Furthermore, the ReLU increased the nonlinear properties of the used model for all networks, without any effect on the receptive fields in the convolution.

\subsection{Pooling layers}

The pooling layer diminishes the information size and accepts the multi-scale analysis. The standard pooling processes include the max pooling and the average pooling. These processes are employed for calculating the maximal or the average values in the local area of the image. In the pooling layer, $2 \times 2$ filters are used to calculate the maximum value $[27,30]$.

\subsection{Fully-connected layers}

The last pooling layer is connected to one or many fully connected layers. This layer considers as hyperparameters, like the number of feature maps, number of convolutional layers, and used dataset [26, 27]. The connection between the fully connected layer and the earlier layer is through the neurons. Furthermore, these layers are utilized as the last system layer. These fully connected layers are used as the final network layer, and are concerned within the classification processes, as shown in Figure 1. CNNs are heavy task and required high computational tools to handle them such as GPUs [31-34] and FPGA [23, 35-37].

\section{METHODOLOGY}

\subsection{The types of $\mathrm{CNN}$ architecture}

Numerous sorts of CNN design are created over the recent couple of years. The mostly well-known CNNs are [38]:

a) LeNet-5 is one of the easiest systems to use. It has two convolutional layers and three fully connected ones. The average-pooling layer was considered as the sub-sampling layer and had a trainable weight. This design has about 60,000 parameters.

b) AlexNet. It is the first successful computer vision CNN. The name comes from Alex Krizhevsky, the main producer. The other two Alex team members are Geoff Hinton and Ilya Sutskever. The ImageNet ILSVRC was questioned in 2012. AlexNet achieved an error of $16 \%$, which is much better than $26 \%$ error of the second-best. AlexNet is the best. It is bigger and deeper, still the same as the LeNet. Furthermore, the featured CONV layers are packed upside down, whereas in LeNet, just single CONV layer is preceded by the pooling layer [39].

c) VGGNet. Karen Simonyan and Andrew Zisserman produced the VGGNet. In the 2014 ILSVRC, it got the second strongest winner. It has revealed that the network depth is a vital component of high-quality results. The absolute highest possible VGGNet has sixteen CONV/FC layers with appealing functionality 
of an extremely reliable design. It just performs $3 \times 3$ convolutions and $2 \times 2$ pooling throughout the entire process. The key weakness of VGGNet is that its measurement is too costly. It also requires large parameters and memory $(140 \mathrm{M})$. And, in the first fully-connected row, a large number of parameters are found. Nevertheless, it was noted that the efficiency of the network is not impacted by the elimination of these fully connected layers [40]. Thus, it is significant to decrease the number of the required parameters.

d) GoogLeNet.: Szegedy et al., from Google developed the GoogLeNet, which was the winner of the 2014 ILSVRC. The key benefit is to make the reduction of network variables (4M) relative to AlexNet's 60M by designing the Inception Module. A further function of GooLeNet is the average pooling. It is employed to decrease a huge range of parameters.

e) ResNet, It was created by Kaiming He et al. The extensive use of batch normalization and the unique skip links are the main features of ResNet. Furthermore, the design of the network requires the fully connected layers as the final stage of the network. At current, ResNets is far away from CNN models [41].

\section{EXPERIENTIAL RESULT AND DISCUSSION}

The four deep learning models (AlexNet, VGG-16, GoogleNet, and ResNet) are implemented in MATLAB2019a on laptop having specification of 32 gigabyte RAM and Intel Core i7 H-Series CPUs (6-Core, 9MB Cache, up to 3.9GHz w/ Turbo Boost).

\subsection{Dataset and pre-processing}

The dataset was collected from different online available resources [42-44]. Three thousand MRI brain tumor images were selected to train the models in two classes: normal and abnormal. The dataset has two folders: yes and no, which includes 3000 images from Brain MRI. The folder yes contains 1800 tumorous (malignant) Brain MRI images, and the folder no contains 1200 non-tumorous (benign) Brain MRI images. This means that 60 percent of the data (1800 images) are positive examples, and 40 percent (1200 images) are negative ones.

\subsection{Data preprocessing}

The following pre-processing procedures have been used for each image:

a) Separating the brain area from the image, which represents the area of importance and interest.

b) Resizing the separated brain image to the form $(240,240,3)$, because the data set has images in different dimensions (width, height, number of channels). All images should have the same shape as a CNN input.

c) Normalizing the images into a scale to $0-1$-pixel values.

\subsection{Data categorize}

Dataset was divided into three groups for training (80\%), validating (10\%), and testing (10\%).

\subsection{Deep learning models}

In deep learning networks, the recent commonly used models are AlexNet, VGG16, GoogLeNet, and ResNet50. These models are employed in this comparative study to classify brain tumors into normal or abnormal. Herein, we tried to compare with four deep learning models in terms of accuracy and time of processing to obtain the best model. The aim of our study is for comparison among AlexNet, VGG16, GoogleNet, and RestNet50. As shown in Figure 2, we took a different MRI brain scan to classify the images into normal and abnormal classes. It is worth mentioning the training cycle has 50 epochs with 1000 iterations and 0.0003 learning rate. The outcomes indicate that the ResNet model has the best performance among the others with an accuracy of $95.8 \%$. In contrast, the AlexNet model has the lowest accuracy of $82.7 \%$.

The second parameter used for comparison is the processing (executing) time, as clarified in Tabel 1. The AlexNet is the fastest one with a processing time of 1.2 seconds, while the slowest model is ResNet with a processing time of 1.9 seconds. The results clearly show that the first parameter (accuracy) behavior is directly proportional with the second parameter (processing time). It means that when the accuracy increases (i.e. more accurate), the processing time also increases (i.e. slow performance). However, to boost the processing time further to be suitable for real-time purposes, a hardware parallel unit named GPU (graphical processing unit) is replaced the general-purpose unit called CPU (central processing unit). This hardware enhances the processing time by more than one hundred times. The AlexNet processing time becomes $8.3 \mathrm{msec}$ (i.e. it is 144 times faster), and the ResNet processing time becomes $29.9 \mathrm{msec}$ (i.e. it is 63 times faster). Table 1 shown the accuracy percentage of CNN models. ResNet has the best percentage because as the deepness increases, the network precision is also increasing, so long as it takes care of over-fitting. 


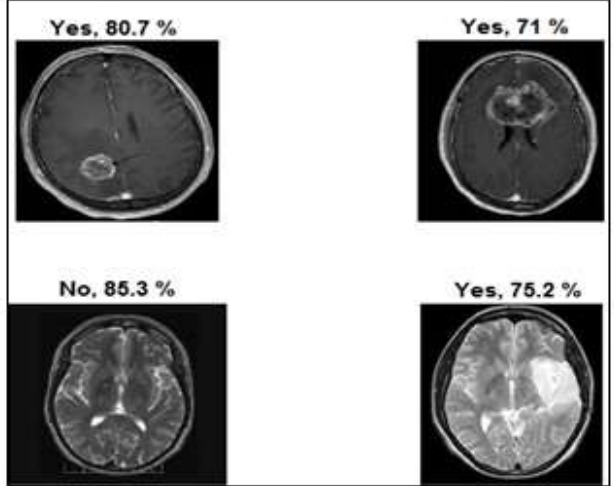

(a)

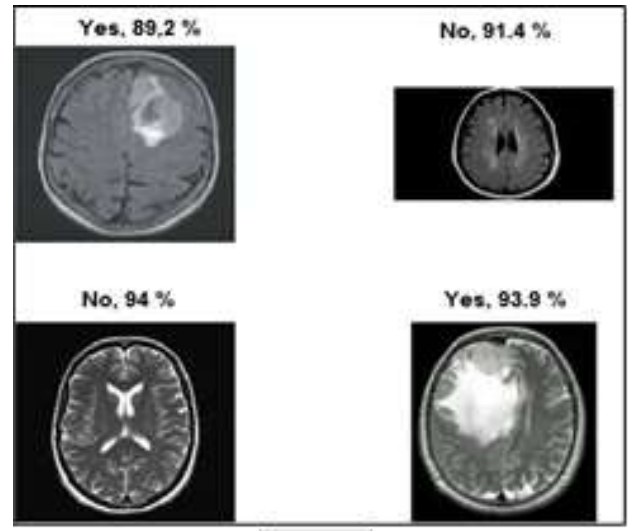

(c)

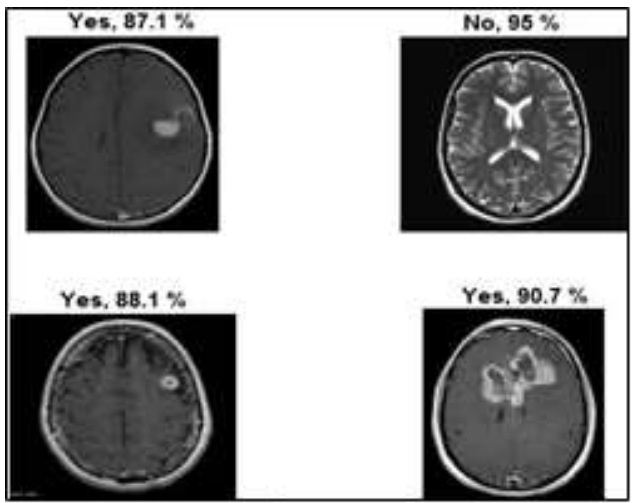

(b)

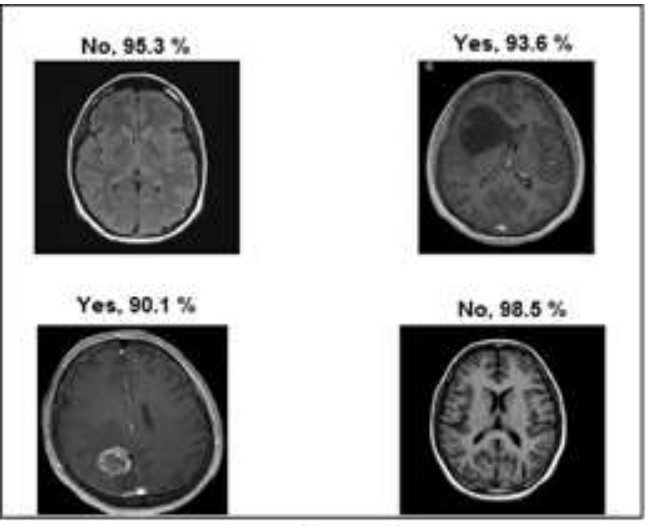

(d)

Figure 2. MRI images for (A) AlexNet model, (B) VGG-16 model, (C) GoogleNet model, (D) ResNet model

Table 1. Comparison between different models of deep learning

\begin{tabular}{|c|c|c|c|c|c|}
\hline \multirow{2}{*}{ Name of Models } & \multirow{2}{*}{ Accuracy } & \multicolumn{2}{|c|}{ Training time } & \multicolumn{2}{|c|}{ Testing Time } \\
\hline & & GPU & $\mathrm{CPU}$ & GPU & CPU \\
\hline AlexNet & 82.7 & $11 \mathrm{~min}$ & $75 \min$ & $8.3 \mathrm{msec}$ & $1.2 \mathrm{sec}$ \\
\hline VGG16 & 86.4 & $24 \mathrm{~min}$ & $93 \min$ & $10.1 \mathrm{msec}$ & $1.5 \mathrm{sec}$ \\
\hline GoogleNet & 91 & $35 \mathrm{~min}$ & $117 \mathrm{~min}$ & $23.7 \mathrm{msec}$ & $1.7 \mathrm{sec}$ \\
\hline ResNet & 95.8 & $40 \mathrm{~min}$ & $165 \mathrm{~min}$ & $29.9 \mathrm{msec}$ & $2 \mathrm{sec}$ \\
\hline
\end{tabular}

\section{CONCLUSION}

The main objectives of this study are to compare performance of four commonly used deep learning models in terms of accuracy and processing time in order to obtain the best model. These models are AlexNet, VGG16, GoogleNet, and RestNet50. The findings of the study showed that ResNet has the highest accuracy, but it is the slowest one. In contrast, AlexNet is the fastest model, but with the lowest accuracy. However, as the depth of the model increases, the accuracy of the network improves; as long as, it takes into account the overfitting issue. Such models are training and testing on general-purpose processor (CPU). The processing time ranged between 1.2 to 1.9 seconds. A hardware parallel unit (GPU) is employed to accelerate the model performance. The boosted performance is ranged from 63 to 144 times. Thus, these models become more appropriate for real-time purposes.

\section{REFERENCES}

[1] M. G. Ertosun and D. L. Rubin, "Automated Grading of Gliomas using Deep Learning in Digital Pathology Images: A modular approach with the ensemble of convolutional neural networks," AMIA Annu. Symp. proceedings. AMIA Symp., vol. 2015, pp. 1899-1908, 2015.

[2] W. H. Ibrahim, A. A. A. Osman, and Y. I. Mohamed, "MRI brain image classification using neural networks," Proc. - 2013 Int. Conf. Comput. Electr. Electron. Eng.' Research Makes a Differ. ICCEEE 2013, pp. 253-258, 2013, doi: 10.1109/ICCEEE.2013.6633943. 
[3] A. Işin, C. Direkoğlu, and M. Şah, "Review of MRI-based Brain Tumor Image Segmentation Using Deep Learning Methods," Procedia Comput. Sci., vol. 102, no. August, pp. 317-324, 2016, doi: 10.1016/j.procs.2016.09.407.

[4] H. Mohsen, E.-S. A. El-Dahshan, E.-S. M. El-Horbaty, and A.-B. M. Salem, "Classification using deep learning neural networks for brain tumors," Futur. Comput. Informatics J., vol. 3, no. 1, pp. 68-71, 2018, doi: 10.1016/j.fcij.2017.12.001.

[5] C. C. Benson, V. L. Lajish, and K. Rajamani, "Brain tumor extraction from MRI brain images using a marker-based watershed algorithm," 2015 Int. Conf. Adv. Comput. Commun. Informatics, ICACCI 2015, pp. 318-323, 2015, doi: 10.1109/ICACCI.2015.7275628.

[6] A. Al-Badarneh, H. Najadat, and A. M. Alraziqi, "A classifier to detect tumor disease in MRI brain images," Proc. 2012 IEEE/ACM Int. Conf. Adv. Soc. Networks Anal. Mining, ASONAM 2012, pp. 784-787, 2012, doi: 10.1109/ASONAM.2012.142.

[7] Y. Zhang, Z. Dong, L. Wu, and S. Wang, "A hybrid method for MRI brain image classification," Expert Syst. Appl., vol. 38, no. 8, pp. 10049-10053, 2011, doi: 10.1016/j.eswa.2011.02.012.

[8] E. S. A. El-Dahshan, T. Hosny, and A. B. M. Salem, "Hybrid intelligent techniques for MRI brain images classification," Digit. Signal Process. A Rev. J., vol. 20, no. 2, pp. 433-441, 2010, doi: 10.1016/j.dsp.2009.07.002.

[9] M. Mohamed Sathik and E. Synthiya Judith Gnanaselvi, "An efficient brain tumor classification based on sobs method for MRI brain images," Int. J. Eng. Adv. Technol., vol. 9, no. 1, pp. 826-833, 2019, doi: 10.35940/ijeat.a9379.109119.

[10] M. Pareek, C. K. Jha, and S. Mukherjee, "Brain Tumor Classification from MRI Images and Calculation of Tumor Area,", vol. 1053, pp. 73-83, 2020, doi: 10.35940/ijeat.a9379.109119.

[11] Atat, Rachad, Lingjia Liu, Jinsong Wu, Guangyu Li, Chunxuan Ye, and Yi Yang, "Big data meet cyber-physical systems: A panoramic survey," IEEE Access, vol. 6, pp. 73603-73636, 2018, doi: 10.1109/ACCESS.2018.2878681.

[12] M. F. Bin Othman, N. Abdullah, and N. A. B. A. Rusli, "An overview of MRI brain classification using FPGA implementation," ISIEA 2010-2010 IEEE Symp. Ind. Electron. Appl., no. Isiea, pp. 623-628, 2010, doi: 10.1109/ISIEA.2010.5679389.

[13] G. Latif, S. B. Kazmi, M. A. Jaffar, and A. M. Mirza, "Classification and Segmentation of Brain Tumor Using Texture Analysis," Recent Adv. Artif. Intell. Knowl. Eng. Data Bases, pp. 147-155, 2010.

[14] A. Kharrat, M. Ben Messaoud, M. Abid, K. Gasmi, and N. Benamrane, "Automated classification of Magnetic Resonance brain images using Wavelet Genetic Algorithm and Support Vector Machine," Proc. 9th IEEE Int. Conf. Cogn. Informatics, ICCI 2010, pp. 369-374, 2010, doi: 10.1109/COGINF.2010.5599712.

[15] V. Sheejakumari and B. Sankara Gomathi, "MRI brain images healthy and pathological tissue classification with the aid of improved particle swarm optimization and neural network," Comput. Math. Methods Med., vol. 2015, 2015, doi: $10.1155 / 2015 / 807826$.

[16] A. Ahmadvand, M. Sharififar, and M. R. Daliri, "Supervised segmentation of MRI brain images using a combination of multiple classifiers," Australas. Phys. Eng. Sci. Med., vol. 38, no. 2, pp. 241-253, 2015, doi: 10.1007/s13246-0150352-7.

[17] C. Saha and M. F. Hossain, "MRI brain tumor images classification using K-means clustering, NSCT and SVM," 2017 4th IEEE Uttar Pradesh Sect. Int. Conf. Electr. Comput. Electron. UPCON 2017, vol. 2018-January, pp. 329333, 2017, doi: 10.1109/UPCON.2017.8251069.

[18] E. I. Zacharaki et al., "Classification of brain tumor type and grade using MRI texture and shape in a machine learning scheme," Magn. Reson. Med., vol. 62, no. 6, pp. 1609-1618, 2009, doi: 10.1002/mrm.22147.

[19] F. Shi, Y. Fan, S. Tang, J. H. Gilmore, W. Lin, and D. Shen, "Neonatal brain image segmentation in longitudinal MRI studies," Neuroimage, vol. 49, no. 1, pp. 391-400, 2010, doi: 10.1016/j.neuroimage.2009.07.066.

[20] Alzubaidi, L., Al-Amidie, M., Al-Asadi, A., Humaidi, A. J., Al-Shamma, O., Fadhel, M. A., ... \& Duan, Y. (2021). Novel Transfer Learning Approach for Medical Imaging with Limited Labeled Data. Cancers, 13(7), 1590.

[21] L. Alzubaidi, Al-Shamma O, Fadhel M. A, Farhan L, Zhang J, "Classification of red blood cells in sickle cell anemia using deep convolutional neural network," In International Conference on Intelligent Systems Design and Applications, pp. 550-559, Springer, Cham, Dec 6 2018, doi: 10.1007/978-3-030-16657-1_51.

[22] R. I. Hasan, Yusuf S. M, Alzubaidi L, "Review of the State of the Art of Deep Learning for Plant Diseases: A Broad Analysis and Discussion," Plant, vol. 9, no. 10, p. 1302, 2020 Oct, doi: 10.3390/plants9101302.

[23] O. Al-Shamma, Fadhel, M. A, Hameed, R. A, Alzubaidi, L, and Zhang, J, "Boosting convolutional neural networks performance based on FPGA accelerator," In International Conference on Intelligent Systems Design and Applications, Springer, Cham, pp. 509-517, December 2018, doi: 10.1007/978-3-030-16657-1_47.

[24] L. Alzubaidi, Al-Shamma O, Fadhel M. A, Arkah Z. M, Awad F. H, "A Deep Convolutional Neural Network Model for Multi-class Fruits Classification," In International Conference on Intelligent Systems Design and Applications, pp. 90-99, Springer, Cham, Dec 3 2019, doi: 10.1007/978-3-030-49342-4_9.

[25] L. a Gatys, A. S. Ecker, and M. Bethge, "A Neural Algorithm of Artistic Style," arXiv Prepr., pp. 1-16, 2015, doi: 10.1167/16.12.326.

[26] Chen, Yu-Hsin and Krishna, Tushar and Emer, Joel, and Sze, Vivienne, "Eyeriss: An Energy-Efficient Reconfigurable Accelerator for Deep Convolutional Neural Networks," in IEEE International Solid-State Circuits Conference, ISSCC 2016, Digest of Technical Papers, pp. 262-263, 2016, doi: 10.1109/JSSC.2016.2616357.

[27] C. Angermueller, T. Pärnamaa, L. Parts, and O. Stegle, "Deep learning for computational biology," Mol. Syst. Biol., vol. 12, no. 7, pp. 1-16, 2016, doi: 10.15252/msb.20156651.

[28] V. Nair and G. E. Hinton, "Rectified Linear Units Improve Restricted Boltzmann Machines," Proc. 27th Int. Conf. Mach. Learn., no. 3, pp. 807-814, 2010. 
[29] G. E. Dahl, T. N. Sainath, and G. E. Hinton, "Improving Deep Neural Networks for \{LVCSR \} Using Rectified Linear Units and Dropout," IEEE Int. Conf. Acoust. Speech Signal Process., pp. 8609-8613, 2013, doi: 10.1109/ICASSP.2013.6639346.

[30] A. Krizhevsky, I. Sutskever, and G. E. Hinton, "ImageNet Classification with Deep Convolutional Neural Networks," Adv. Neural Inf. Process. Syst., pp. 1-9, 2012, doi: 10.1145/3065386.

[31] L.Alzubaidi, Fadhel, M. A., Al-Shamma, O., Zhang, J., and Duan, Y, "Deep Learning Models for Classification of Red Blood Cells in Microscopy Images to Aid in Sickle Cell Anemia Diagnosis," Electronics, vol. 9, no. 3, p. 427, 2020, doi: 10.3390/electronics9030427.

[32] L.Alzubaidi, Al-Shamma, O., Fadhel, M. A., Farhan, L., Zhang, J., and Duan, Y, "Optimizing the performance of breast cancer classification by employing the same domain transfer learning from hybrid deep convolutional neural network model", Electronics, vol. 9,no. 3, pp. 445, 2020, doi: 10.3390/electronics9030445.

[33] L. Alzubaidi, Fadhel, M. A, Oleiwi, S. R, Al-Shamma, O, and Zhang, J, "DFU_QUTNet: diabetic foot ulcer classification using novel deep convolutional neural network," Multimedia Tools and Applications, pp. 1-23, 2019, doi: 10.1007/s11042-019-07820-w.

[34] L. Alzubaidi, Fadhel M. A, Al-Shamma O, Zhang J, Santamaría J, Duan Y, Oleiwi S. R, "Towards a better understanding of transfer learning for medical imaging: a case study," Applied Sciences, vol. 10, no. 13, p. 4523, 2020 Jan, doi: 10.3390/app10134523.

[35] Fadhel, M. A, Al-Shamma, O, Oleiwi, S. R, Taher, B. H, and Alzubaidi, L, "Real-time PCG diagnosis using FPGA," In International Conference on Intelligent Systems Design and Applications, pp. 518-529, Springer, Cham, 2018, December, doi: 10.1007/978-3-030-16657-1_48.

[36] Fadhel, M. A, Al-Shamma, O, Alzubaidi, L, and Oleiwi, S. R, "Real-Time Sickle Cell Anemia Diagnosis Based Hardware Accelerator," In International Conference on New Trends in Information and Communications Technology Applications, pp. 189-199, Springer, Cham, 2020, June, doi: 10.1007/978-3-030-55340-1_14.

[37] Fadhel, M. A, Al-Shamma, O, and Alzubaidi, L, "Hardware Accelerator for Real-Time Holographic Projector," In International Conference on Intelligent Systems Design and Applications, pp. 132-139, Springer, Cham, 2019, December, doi: 10.1007/978-3-030-49342-4_13.

[38] Alzubaidi, L., Zhang, J., Humaidi, A. J., Al-Dujaili, A., Duan, Y., Al-Shamma, O., ... \& Farhan, L. (2021). Review of deep learning: concepts, CNN architectures, challenges, applications, future directions. Journal of Big Data, 8(1), $1-74$.

[39] J. Cong and B. Xiao, "Minimizing Computation in Convolutional Neural Networks," Int. Conf. Artif. Neural Networks, pp. 281-290, 2014, doi: 10.1007/978-3-319-11179-7_36.

[40] J. Xu, T. Mei, T. Yao, and Y. Rui, "MSR-VTT: A large video description dataset for bridging video and language," Proc. IEEE Comput. Soc. Conf. Comput. Vis. Pattern Recognit., vol. 2016-December, pp. 5288-5296, 2016.

[41] M. Rastegari, V. Ordonez, J. Redmon, and A. Farhadi, "XNOR-net: Imagenet classification using binary convolutional neural networks," Lect. Notes Comput. Sci. (including Subser. Lect. Notes Artif. Intell. Lect. Notes Bioinformatics), vol. 9908 LNCS, pp. 525-542, 2016, doi: 10.1007/978-3-319-46493-0_32.

[42] Kaggle: https://www.kaggle.com/ruslank1/brain-tumor-detection-v1-0-cnn-vgg-16.

[43] Github: https://github.com/MohamedAliHabib/Brain-Tumor-Detection.

[44] Brain tumor: https://braintumor.org/brain-tumor-information/. 